\title{
Errata: On the Rate of Convergence to the Normal Law for Solutions of the Burgers Equation with Singular Initial Data ${ }^{1}$
}

\author{
N. N. Leonenko, E. Orsingher, and V. N. Parkhomenko
}

Received February 22, 1996

The following minor mistakes in our paper should be corrected. On page 919, Eq. (27),

$$
X(a)=-\frac{1}{i}\left[\frac{2}{\Gamma(\alpha+1) \cos (\alpha \Pi / 2)}\right]^{1 / 2} \int_{-\infty}^{+\infty} e^{i \lambda a} g(\lambda) W(d \lambda)
$$

should be replaced by

$$
X(a)=-\frac{1}{i}\left[\frac{\alpha / 2}{\Gamma(\alpha+1) \cos (\alpha \Pi / 2)}\right]^{1 / 2} \int_{-\infty}^{+\infty} e^{i \lambda a} g(\lambda) W(d \lambda)
$$

On p. 919, Eq. (3.1),

$$
\Delta_{t}=\sup _{-\infty<z<+\infty}\left|P\left\{\frac{1}{\sigma} \tilde{X}_{t}(a) \leqslant z\right\}-\Phi(z)\right|, \quad \sigma_{2}=R(a, a)
$$

and the subsequent "where $\widetilde{X}_{t}(a), a \in \mathbb{R}^{1}$, is defined by $(2.5), \Phi(z)$ is defined by (2.4), and $R(a, b)$ is defined by (2.6)," should be replaced by

$$
\Delta_{t}=\sup \left|P\left\{\frac{1}{\sigma} \tilde{X}_{t}(a) \leqslant z\right\}-\Phi(z)\right|
$$

where $\tilde{X}_{l}(a), a \in \mathbb{R}^{1}$, is defined by $(2.5), \Phi(z)$ is defined by $(2.4)$, and

$$
\begin{gathered}
\sigma^{2}=\sigma_{t}^{2}=(2 \mu)^{-1-(\alpha / 2)} \int_{A(a, t)} \int_{A(a, t)} \frac{w_{1} w_{2} \phi\left(w_{1}\right) \phi\left(w_{2}\right) L\left(\left|w_{1}-w_{2}\right| \sqrt{2 \mu t}\right)}{\left|w_{1}-w_{2}\right|^{\alpha} L(\sqrt{t})} d w_{1} d w_{2} \\
A(a, t)=\left[\frac{a}{(2 \mu)^{1 / 2}}-\left(\frac{t}{2 \mu}\right)^{1 / 2}, \frac{a}{(2 \mu)^{1 / 2}}+\left(\frac{t}{2 \mu}\right)^{1 / 2}\right]
\end{gathered}
$$

\footnotetext{
' This paper appeared in J. Stat. Phys. 82(3/4):915-930 (1996).
} 
On p. 924 , the find line of (3.9), instead of

$$
=\frac{L(\sqrt{t})}{t^{\alpha / 2}} K_{t} \frac{1}{(\sqrt{2 \mu})^{\alpha / 2} C_{1}^{2}} \sum_{k=2}^{\infty} \frac{C_{k}^{2}}{k !}
$$

one should have

$$
=\frac{L(\sqrt{t})}{t^{\alpha / 2}} K_{t} \frac{1}{(2 \mu)^{\alpha / 2} C_{1}^{2}} \sum_{k=2}^{\infty} \frac{C_{k}^{2}}{k !}
$$

On p. 925, line 4, instead of

$$
\times\left[\int_{-\infty}^{\infty} \int_{-\infty}^{\infty} w_{1} w_{2} \varphi\left(w_{1}\right) \varphi\left(w_{2}\right) \frac{d w_{1} d w_{2}}{\left|w_{1}-w_{2}\right|^{\alpha}}\right]^{-1}
$$

one should have

$$
\times\left[\int_{A(a, t)} \int_{A(a, t)} w_{1} w_{2} \varphi\left(w_{1}\right) \varphi\left(w_{2}\right) \frac{L\left(\left|w_{1}-w_{2}\right| \sqrt{2 \mu t}\right)}{L(\sqrt{t})} \frac{d w_{1} d w_{2}}{\left|w_{1}-w_{2}\right|^{\alpha}}\right]^{-1}
$$

On p. 928, the second line of (3.20), instead of

$$
\leqslant \frac{1}{\varepsilon^{2}}\left\{\frac{\theta^{2} M_{t}(\alpha) L(\sqrt{t})}{t^{\alpha / 2}}+c_{4} Q_{r}\right\}, \quad c_{4}=\text { const }>0
$$

one should have

$$
\leqslant \frac{1}{\varepsilon^{2}}\left\{\frac{\theta^{2} M_{t}(\alpha) L(\sqrt{t})}{t^{\alpha / 2+2}(2 \mu)^{\alpha / 2}}+c_{4} Q_{t}\right\}, \quad c_{4}=\text { const }>0
$$

On p. 928 , line 10 , instead of

$$
\varepsilon=\frac{L^{1 / 3}(\sqrt{t})}{t^{\alpha / 6}}\left(1+\frac{1}{\sqrt{2}}\right)^{-1 / 3}(2 \mu)^{-\alpha / 6}\left(2 c_{2} K_{t}+\frac{M_{1}(\alpha)}{2 \mu^{2}}\right)^{1 / 3}
$$

one should have

$$
\varepsilon=\frac{L^{1 / 3}(\sqrt{t})}{t^{\alpha / 6}}\left(1+\frac{1}{\sqrt{2 \pi}}\right)^{-1 / 3}(2 \mu)^{-\alpha / 6} \theta^{2 / 3}\left(2 c_{2} K_{t}+\frac{M_{t}(\alpha)}{2 \mu^{2}}\right)^{1 / 3}
$$

On p. 928 , line 12 , instead of

$$
\Delta_{1} \leqslant \frac{L^{1 / 3}(\sqrt{t})}{t^{\alpha / 6}}\left[v_{1}^{2 / 3} v_{2}^{1 / 3}+\frac{v_{1}^{2 / 3} v_{2}^{1 / 3}}{2}+\frac{t^{\alpha / 2}}{L(\sqrt{t})}\left(R_{t} c_{5}+c_{6} Q_{t}\right)\right]
$$




\section{Errata}

one should have

$$
\Delta, \leqslant \frac{L^{1 / 3}(\sqrt{t})}{t^{\alpha / 6}}\left[v_{1}^{2 / 3} \tilde{v}_{2}^{1 / 3}+\frac{v_{1}^{2 / 3} \tilde{v}_{2}^{1 / 3}}{2}+\frac{t^{\alpha / 2}}{L(\sqrt{t})}\left(R_{t} c_{5}+c_{6} Q_{t}\right)\right]
$$

where

$$
\tilde{v}_{2}=\theta^{2}(2 \mu)^{2-x / 2}\left\{2 K_{t}\left[e^{1 /\left(2 \mu^{2}\right)}-\left(1+\frac{1}{4 \mu^{2}}\right) e^{1 /\left(4 \mu^{2}\right)}\right] e^{-1 /\left(4 \mu^{2}\right)}+\frac{M_{t}(\alpha)}{8 \mu^{4}}\right\}
$$

